\title{
Regulation of IGF-I Signaling in Retinal Endothelial Cells by Hyperglycemia
}

\author{
Emily C. Miller, Byron E. Capps, Ravi R. Sanghani, David R. Clemmons, and \\ Laura A. Maile
}

Purpose. To investigate the role of hyperglycemia in regulating the proliferative response of retinal endothelial cells (RECs) to insulin-like growth factor (IGF)-I.

Methods. The regulation of IGF-I signaling by glucose concentration was assessed by biochemical analysis of primary RECs grown in media containing normal $(5 \mathrm{mM})$ and high $(25 \mathrm{mM})$ glucose. Cell counting was used to asses the proliferative response to IGF-I.

Results. Glucose (25 mM) enhanced the proliferative response of RECs to IGF-I. Phosphorylation of the adaptor protein Shc (Src homology 2 domain containing) transforming protein 1) was increased in RECs grown in high glucose. For Shc to be phosphorylated, it must be recruited to the cytoplasmic domain of the transmembrane protein SHPS-1 (SHP substrate-1). Shc recruitment to SHPS-1 was increased when RECs were grown in high glucose. The difference in Shc recruitment to SHPS-1 was attributable to a difference in SHPS-1 phosphorylation that is required for Shc recruitment. This, in turn, was attributable to an increase in SHPS-1 association with integrinassociated protein (IAP), which is necessary for SHPS-1 phosphorylation. The difference in response under the two different glucose conditions appeared to be attributable to changes in the activation of the integrin $\alpha \mathrm{V} \beta 3$, since blocking $\alpha \mathrm{V} \beta 3$ in high glucose inhibited the response to IGF-I, whereas addition of the active region of vitronectin to RECs grown in normal glucose enhanced their response.

Conclusions. This study demonstrates that hyperglycemic conditions enhance the response of RECs to IGF-I by increasing the association of IAP with SHPS-1 permitting the formation of the SHPS-1-Shc signaling complex, which is required for the proliferative response to IGF-I. (Invest Ophthalmol Vis Sci. 2007; 48:3878-3887) DOI:10.1167/iovs.07-0014

$\mathrm{T}$ he most common cause of blindness in patients with type 1 diabetes is proliferative diabetic retinopathy (PDR) with the growth of unwanted blood vessels and intravitreous neovascularization (IVNV). ${ }^{1}$ Formation of these new blood vessels requires retinal endothelial cell (REC) proliferation and migration. ${ }^{2}$ Hyperglycemia appears to contribute directly to the neovascularization associated with PDR, since large clinical trials have shown that tight glucose control in diabetic patients

From the Division of Endocrinology, Department of Medicine, University of North Carolina at Chapel Hill, Chapel Hill, North Carolina. Supported by an American Diabetes Association Junior Faculty Award (LAM).

Submitted for publication January 5, 2007; revised March 12, 2007; accepted May 11, 2007.

Disclosure: E.C. Miller, None; B.E. Capps, None; R.R. Sanghani, None; D.R. Clemmons, Genentech (F); L.A. Maile, None

The publication costs of this article were defrayed in part by page charge payment. This article must therefore be marked "advertisement" in accordance with 18 U.S.C. $\$ 1734$ solely to indicate this fact.

Corresponding author: Laura A. Maile, Division of Endocrinology, Department of Medicine, University of North Carolina at Chapel Hill, Chapel Hill, NC 27599-7170; laura_maile@med.unc.edu. reduces the progression of this disease. ${ }^{3}$ Achieving and maintaining such tight glucose control is challenging, and retina ablation is currently the only therapy for direct treatment of PDR.

There is a significant amount of data to suggest that increases in insulin-like growth factor (IGF)-I bioactivity may contribute to retinal neovascularization, characteristic of conditions such as proliferative diabetic retinopathy and retinopathy of prematurity. ${ }^{4,5}$ Retinal endothelial cells (RECs) express both IGF-I and IGF-I receptors (IGF-IRs). In a mouse model of oxygen-induced retinopathy it was shown that IGF-IR antagonists suppressed retinal neovascularization. ${ }^{4}$ In a similar model, it has been shown that endothelial cell-specific knockout of the IGF-IR (and insulin receptor) protects against neovascularization. ${ }^{5}$ There is some evidence to suggest that the effect of IGF-I is mediated at least in part by its ability to control vascular endothelial cell growth factor stimulated mitogen-activated protein kinase (MAPK) activation. ${ }^{4}$ Although inhibiting IGF-I activity would seem like an attractive strategy for reducing neovascularization in these conditions, directly inhibiting IGF-I, or its receptor, is unlikely to be a viable approach, because this approach is likely to be associated with significant toxicity. Although inhibiting IGF-I signaling in endothelial cells is desirable, inhibiting IGF-I signaling in neurons, for example, where it is an important survival factor, would be undesirable. ${ }^{6}$

We reasoned that identification of factors that specifically regulate the response of RECs to IGF-I may provide novel, cell-type-specific targets for the inhibition of IGF-I signaling. $\alpha \mathrm{V} \beta 3$ integrin is a marker of proliferating endothelial cells and $\alpha \mathrm{V} \beta 3$ antagonists have been shown to inhibit both retinal and tumor angiogenesis. ${ }^{7-13}$ We have shown in smooth muscle cells (SMCs) that $\alpha \mathrm{V} \beta 3$ ligand occupancy is necessary for the positive effects of IGF-I on these cells. We have shown in SMCs that binding of the heparin-binding domain (HBD) of the principal $\alpha \mathrm{V} \beta 3$ ligand vitronectin (Vn) is sufficient for the positive effects of Vn on IGF-I signaling in these cells. Furthermore, it is an interaction between a cysteine loop region (C-loop region) of the extracellular region of $\beta 3$ (between amino acids 177 184) and the Vn HBD that mediates the effects of $\mathrm{Vn} .{ }^{14,15}$ When binding between the Vn HBD and the C-loop region is blocked in the presence of an antibody raised specifically against the C-loop region of $\beta 3$, IGF-I stimulated migration and proliferation of SMCs is inhibited. ${ }^{15}$ The interaction between the Vn HBD and the C-loop region of $\beta 3$ is distinct from the interaction between the RGD sequence of the integrin ligand and $\alpha \mathrm{V} \beta 3$. Previous studies have suggested that changes in levels of $\alpha \mathrm{V} \beta 3$ ligands may occur in response to hyperglycemia, ${ }^{16-21}$ and we hypothesized that increases in $\alpha \mathrm{V} \beta 3$ ligand occupancy, under conditions of hyperglycemia, may contribute to enhanced REC proliferation, and therefore the development of PDR, by increasing the proliferative response to IGF-I.

An understanding of the molecular events that regulate the proliferation of RECs leading to PDR is essential for the identification of therapeutic targets and strategies. The purpose of this study was to determine whether hyperglycemia-induced changes in $\alpha \mathrm{V} \beta 3$ ligand occupancy regulate the proliferative 
response of RECs to IGF-I and to determine the molecular mechanism by which this regulation occurs.

\section{Materials AND Methods}

Human IGF-I was a gift from Genentech (South San Francisco, CA). Polyvinyl difluoride membranes (Immobilon P) were purchased from Millipore Corp. (Billerica, MA). Autoradiographic film was obtained from Pierce (Rockford, IL). The IGF-IR $\beta$ chain polyclonal and the monoclonal phosphotyrosine antibody (PY99) were purchased from Santa Cruz Biotechnology (Santa Cruz, CA). The Shc, phospho/total ERK1/2 and SHPS-1 antibodies were purchased from BD Biosciences (San Jose, CA). The anti-IAP monoclonal antibody, B6H12, was prepared from conditioned medium from a specific B-cell hybridoma line, as we have described previously. ${ }^{22}$ The Vn antibody was prepared as we have described previously. ${ }^{23} \mathrm{~A}$ mouse control immunoglobulin (IgG) was purchased from Roche Applied Sciences (Indianapolis, IN). All other reagents were purchased from Sigma-Aldrich (St. Louis, MO), unless otherwise stated.

\section{Retinal Endothelial Cells}

Primary bovine retinal endothelial cells (RECs) were obtained from VEC Technologies Inc. (Rensselaer, NY). Stock cultures of RECs were grown in a defined EC growth medium (MCDB-131 complete) supplied by VEC Technologies, Inc., which contains glucose at a physiologically normal concentration of $5 \mathrm{mM}$ glucose. Stock cultures of RECs were grown in p100 dishes precoated with $50 \mu \mathrm{g} / \mathrm{mL}$ fibronectin in PBS for 30 minutes at $37^{\circ} \mathrm{C}$.

\section{B3 Antibodies}

A $\beta 3$ antibody specific for the cytoplasmic tail of $\beta 3$, which was used for immunoprecipitation and immunoblotting, was prepared by injecting rabbits with a peptide containing amino acids $742-762$ of $\beta 3$ that had been conjugated to keyhole limpet hemocyanin (KLH). An antibody against the C-loop region of the extracellular domain of $\beta 3$ (C-loop $\beta 3$ ) was prepared by injecting rabbits with a peptide containing amino acids $177-184 .{ }^{15}$ This antibody was used to block the Vn binding to $\beta 3$. RECs were treated with the C-loop $\beta 3$ antibody or control rabbit immunoglobulin (IgG) at a concentration of $1 \mu \mathrm{g} / \mathrm{mL}$.

\section{Protein Purification and Synthetic Peptide Synthesis}

Vn was purified from porcine serum, as we have described previously. ${ }^{24}$ A synthetic peptide corresponding to the heparin-binding domain (HBD) of human Vn (amino acids 365-LAKKQRFRHRNRKGYRS-381) was synthesized and purified as we have described elsewhere. ${ }^{11}$

\section{Cell Proliferation}

RECs that had been plated in medium containing $5 \mathrm{mM}$ glucose were fed 24 hours after plating with medium containing either 5 or $25 \mathrm{mM}$ glucose. The following day, these cells were used to plate $2 \times 10^{4}$ cells per well in each well of a 24 -well plate precoated with fibronectin ( 50 $\mu \mathrm{g} / \mathrm{mL}$ ). The cells were left to attach for 4 hours before the medium was replaced with treatments (anti-IAP monoclonal, B6H12, or control IgG [10 $\mu \mathrm{g} / \mathrm{mL}]$ or C-loop $\beta 3$ antibody or control IgG $[1 \mu \mathrm{g} / \mathrm{mL}]$ or Vn HBD $[5-20 \mu \mathrm{g} / \mathrm{mL}]$ in the presence or absence of IGF-I ([10-100 $\mu \mathrm{g} / \mathrm{mL}])$ prepared in serum-free medium containing either 5 or $25 \mathrm{mM}$ glucose (medium containing $5 \mathrm{mM}$ glucose was supplemented with mannitol to control for differences in osmolarity) plus $0.2 \%$ fetal bovine serum (FBS). After a 48 -hour incubation at $37^{\circ} \mathrm{C}$, cell number was determined after trypsinization, using trypan blue staining and counting. ${ }^{14}$

\section{Cell Lysis, Immunoprecipitation, and Western Immunoblot Analysis}

RECs were plated on dishes coated with $50 \mu \mathrm{g} / \mathrm{mL}$ fibronectin in medium containing $5 \mathrm{mM}$ glucose. The following day, the cells were fed with medium containing either 5 or $25 \mathrm{mM}$ glucose. Forty-eight hours later, when the cell monolayers were approximately $80 \%$ confluent, cells were quiesced overnight in SFM before the addition of IGF-I $(100 \mathrm{ng} / \mathrm{mL})$ for the times indicated. In some experiments, RECs were treated with either the C-loop $\beta 3$ antibody $(1 \mu \mathrm{g} / \mathrm{mL})$ or the anti-IAP monoclonal antibody $\mathrm{B} 6 \mathrm{H} 12(10 \mu \mathrm{g} / \mathrm{mL})$ or appropriate control IgG for 4 hours or Vn $(1 \mu \mathrm{g} / \mathrm{mL})$ or the Vn HBD $(5-20 \mu \mathrm{g} / \mathrm{mL})$ for 2 hours before the addition of IGF-I $(100 \mathrm{ng} / \mathrm{mL})$. Cell monolayers were lyzed with a modified radioimmunoprecipitation buffer. After centrifugation, lysates were used for immunoprecipitation with the appropriate antibody at $4^{\circ} \mathrm{C}$. The proteins were visualized after SDS-PAGE and electrophoretic transfer by using Western immunoblot analysis.

\section{Statistical Analysis}

Chemiluminescent images obtained were scanned (DuoScan T1200; AGFA Brussels, Belgium), and band intensities of the scanned images were analyzed using NIH Image, version 1.61 (available by $\mathrm{ftp}$ at zippy.nimh.nih.gov/ or at http://rsb.info.nih.gov/nih-image; developed by Wayne Rasband, National Institutes of Health, Bethesda, MD). The Student's $t$-test was used to compare differences between treatments. The results that are shown in all experiments are representative of at least three separate experiments.

\section{Results}

\section{Hyperglycemia Enhances the Proliferative Response of RECs to IGF-I}

We determined whether glucose levels directly regulated the response of RECs to IGF-I by comparing the proliferative response of RECs grown in medium containing a physiologically normal level of glucose $(5 \mathrm{mM})$ and the response of RECs grown in medium containing a high level of glucose $(25 \mathrm{mM})$. RECs grown in normal glucose did not demonstrate a significant increase (1.18 \pm 0.16-fold; mean \pm SEM, $n=3)$ in proliferation in response to IGF-I. However, RECs that had been cultured in medium containing $25 \mathrm{mM}$ glucose demonstrated a significant $2.2 \pm 0.15$-fold (mean $\pm \mathrm{SEM}, n=3$ ) increase in the number of cells in response to IGF-I (Fig. 1A). Since glucose has been shown to have direct effects (both stimulatory and inhibitory), we examined the effects of increasing concentrations of glucose in our system. Increasing concentrations of glucose had no effect on the proliferation of RECs under these conditions and therefore the difference in response to IGF-I is not due to a direct effect of glucose on REC cell proliferation (data not shown).

We hypothesized that the difference in proliferation between RECs grown in 5 and $25 \mathrm{mM}$ glucose may be due to changes in the activation state of the $\alpha \mathrm{V} \beta 3$ integrin. Consistent with this hypothesis, the enhanced response of RECs in $25 \mathrm{mM}$ glucose was blocked when $\alpha \mathrm{V} \beta 3$ ligand binding was blocked in the presence of the C-loop $\beta 3$ antibody that blocks binding of $\mathrm{Vn}$ to $\beta 3^{15}$ (a $1.0 \pm 0.1$-fold $[n=3$ ] increase in proliferation in the presence of the C-loop $\beta 3$ antibody). The control IgG did not have a significant effect on IGF-I stimulated cell proliferation. Also consistent with our hypothesis, the response of RECs grown in $5 \mathrm{mM}$ glucose was enhanced by the addition of the $\mathrm{Vn}$ HBD (the region of $\mathrm{Vn}$ that we have shown is necessary for the enhancing effects of Vn on IGF-I signaling ${ }^{14,15}$ ). In the presence of the Vn HBD IGF-I stimulated a significant, $2.2 \pm$ 0.25 -fold increase in the number of cells (mean \pm SEM, $n=3$, 




Figure 1. Regulation of IGF-I-stimulated REC proliferation. (A, B) The cells $\left(2 \times 10^{4}\right)$ were plated in each well of a 24-well plate coated with 50 $\mu \mathrm{g} / \mathrm{mL}$ of fibronectin and left to attach in medium containing either 5 or $25 \mathrm{mM}$ glucose. The media were then replaced with serum free medium (containing the appropriate glucose concentration) plus $0.2 \% \mathrm{fe}$ tal bovine serum plus treatments as indicated: IGF-I; (25-100 ng/mL); Cloop $\beta 3$ antibody (C-loop IgG) or control (Con) IgG, both at a concentration of $1 \mu \mathrm{g} / \mathrm{mL}$; or Vn HBD (10 $\mu \mathrm{g} / \mathrm{mL}$ ). Forty-eight hours after the addition of IGF-I the number of cells was determined by trypan blue staining and counting. ${ }^{* * *} P<0.005$, ${ }^{* *} P<0.01$ when the increase in the number of cells in the presence of IGF-I is compared with the increase in the number in the absence of IGF-I.
$P<0.01$; Fig. 1A). To demonstrate that the effect of Vn HBD was mediated through the $\alpha \mathrm{V} \beta 3$ integrin, we determined the ability of the Vn HBD to enhance IGF-I signaling in the presence of the C-loop $\beta 3$ antibody. In the presence of this antibody, the ability of the Vn HBD to enhance IGF-I signaling was completely inhibited (Fig. 1A), whereas control IgG had no effect.

A dose response to the Vn HBD revealed that, when added alone, it had a small, nonsignificant stimulatory effect on REC proliferation. These results suggest that either direct activation of the $\alpha \mathrm{V} \beta 3$ integrin itself is sufficient to stimulate cell proliferation or that activation of the $\alpha \mathrm{V} \beta 3$ integrin is enhancing signaling by endogenously produced IGF-I.

\section{Glucose Regulation of $\boldsymbol{\beta 3}$ Phosphorylation}

Phosphorylation of $\beta 3$ is a marker of its activation state. We compared the $\beta 3$ phosphorylation between RECs grown in 25
$\mathrm{mM}$ glucose compared with $5 \mathrm{mM}$ glucose. There was little detectable $\beta 3$ phosphorylation in RECs grown in $5 \mathrm{mM}$ glucose but there was a significant, $4.1 \pm 0.3$-fold (mean \pm SEM, $n=$ 3 ) increase in $\beta 3$ phosphorylation levels when $\beta 3$ phosphorylation in RECs grown in $25 \mathrm{mM}$ glucose was compared with RECs grown in $5 \mathrm{mM}$ glucose (Fig. 2A). There was no significant difference in the amount of $\beta 3$ protein levels that would account for the difference in $\beta 3$ phosphorylation. However, examination of the amount of the $\alpha \mathrm{V} \beta 3$ ligand, Vn that was associated with $\beta 3$ revealed that there was a $6.3 \pm 2.2$-fold increase in $\mathrm{Vn}$ association with $\beta 3$ in RECs grown in $25 \mathrm{mM}$ glucose compared with those grown in $5 \mathrm{mM}$ glucose (mean \pm SEM, $n=3$ ). Because ligand occupancy of $\beta 3$ regulates its phosphorylation, ${ }^{25}$ this increase presumably accounts for the increase in $\beta 3$ phosphorylation.

To demonstrate the role of Vn HBD binding to the C-loop region of $\beta 3$ in regulating $\beta 3$ phosphorylation, we examined
A

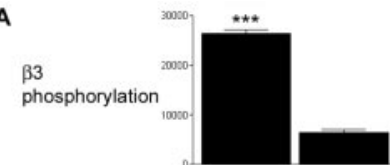

IP: $\beta 3 / 1 B:-p-T y r$

IB: $\beta 3$

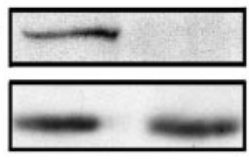

25
B

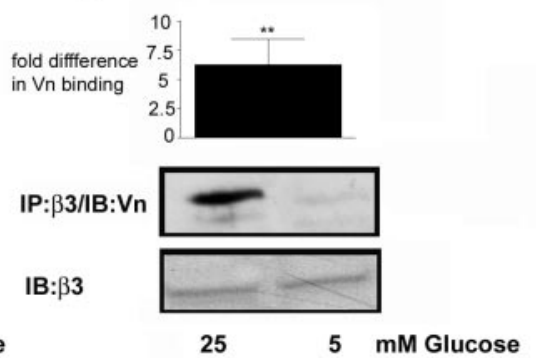

D

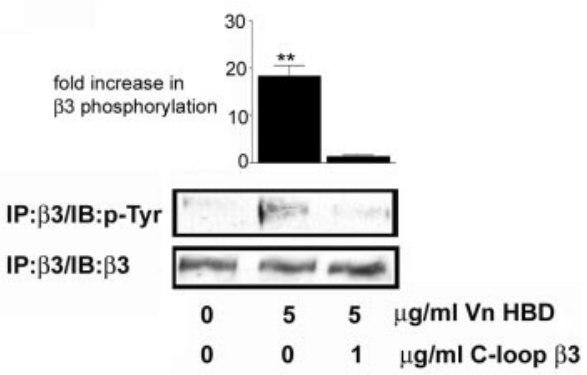

FigURE 2. Glucose regulation of $\beta 3$ protein phosphorylation. RECs were grown in medium containing either 25 or $5 \mathrm{mM}$ glucose and then quiesced overnight in serum-free medium. (A) The extent of $\beta 3$ phosphorylation was determined by immunoprecipitating cell lysates with an anti- $\beta 3$ antibody raised to the extracellular region of $\beta 3$ and then immunoblotting with an anti-phosphotyrosine antibody (p-Tyr). $\beta 3$ protein levels were detected by immunoblotting cell lysates directly with an anti- $\beta 3$ antibody. The graph show the data from three independent experiments expressed as arbitrary scanning units. ${ }^{* * *} P<0.005$ when $\beta 3$ phosphorylation in RECs grown in $25 \mathrm{mM}$ glucose is compared with $5 \mathrm{mM}$ glucose. (B) Vitronectin (Vn) association with $\beta 3$ was determined by immunoprecipitating cell lysates with and anti- $\beta 3$ antibody and immunoblotting with an anti-Vn antibody. $\beta 3$ protein levels were detected by immunoblotting cell lysates directly with an anti $\beta 3$ antibody. ${ }^{* *} P<0.005$ when $\mathrm{Vn}$ association in $\beta 3$ in RECs grown in $25 \mathrm{mM}$ glucose is compared with $\mathrm{Vn}$ association in RECs grown in $5 \mathrm{mM}$. (C, D) Vn heparin binding domain peptide (Vn HBD), intact Vn (at concentrations indicated) or the C-loop $\beta 3$ antibody (C-loop IgG; $1 \mu \mathrm{g} / \mathrm{mL}$ ) were added for 2 hours. The extent of $\beta 3$ phosphorylation was determined by immunoprecipitating cell lysates with an anti- $\beta 3$ antibody and then immunoblotting with an anti-phosphotyrosine antibody (p-Tyr). The graph shows the mean increase in $\beta 3$ phosphorylation with increasing amounts of Vn HBD $(n=3$ independent experiments, ${ }^{* *} P<0.01$ when the increase in the presence of Vn HBD is compared with basal levels of $\beta 3$ phosphorylation). $\beta 3$ protein levels were detected by immunoblotting cell lysates with an antibody to $\beta 3$. 
the effect of adding increasing amounts of Vn HBD to RECs grown in normal glucose. Phosphorylation of $\beta 3$ increased in a dose-dependent manner with the addition of 1,5 , and 10 $\mu \mathrm{g} / \mathrm{mL}$ of $\mathrm{Vn}$ HBD resulting in a $10 \pm 3.5-, 34 \pm 2.1-$, and $46 \pm$ 2 -fold increase, respectively (mean $\pm \mathrm{SEM}, n=3$ ). The increase in $\beta 3$ phosphorylation in the presence of $5 \mu \mathrm{g} / \mathrm{mL}$ (a $10 \pm 1.35$-fold increase [mean $\pm \mathrm{SEM}, n=3$ ]) was similar to the $16 \pm 2$-fold (mean \pm SEM, $n=3$ ) increase seen in response to whole $\mathrm{Vn}(1 \mu \mathrm{g} / \mathrm{mL})$. Direct examination of $\beta 3$ protein levels revealed that there was no significant difference in $\beta 3$ protein levels to account for the difference in $\beta 3$ phosphorylation (Fig. 2C).

In the presence of the C-loop $\beta 3$ antibody, the ability of the Vn HBD to increase $\beta 3$ phosphorylation was completely inhibited (Fig. 2D). Phosphorylation of $\beta 3$ was reduced from a $18 \pm$ 0.2 - to a $1.3 \pm 0.3$-fold increase, compared with basal levels, after the addition of the antibody (differences are expressed as the mean $\pm \mathrm{SEM}, n=3$ ). Control IgG had no effect (data not shown).

\section{Hyperglycemia Regulation of She and SHPS-1 Phosphorylation in Response to IGF-I}

Having determined that hyperglycemia resulted in an increase in REC responsiveness to IGF-I in an $\alpha \mathrm{V} \beta 3$-dependent manner, we wished to determine the mechanism by which the hyperglycemia-induced increase in $\beta 3$ activation regulated the response of RECs to IGF-I. IGF-IR signaling is coupled to downstream signaling events via phosphorylation of the adaptor proteins, insulin-receptor substrates (IRS-1 through -4) or Shc. ${ }^{26}$ We compared Shc phosphorylation in the two different glucose conditions (Fig. 3A). In contrast to the lack of Shc phosphorylation (a $1.1 \pm 0.1$-fold increase; $n=3$ ]) in response to IGF-I when RECs were grown in $5 \mathrm{mM}$ glucose, IGF-I stimulated a significant $(11.8 \pm 2$.8-fold $)$ increase $(n=3)$ in Shc phosphorylation when RECs were grown in $25 \mathrm{mM}$ glucose (Fig. 3A).

Phosphorylation of Shc coupled the IGF-IR to activation of the MAP kinase (MAPK) pathway. Consistent with the difference in Shc phosphorylation, there was a significant difference in the extent of ERK1/2 phosphorylation, a marker of MAPK pathway activation, in response to IGF-I (Fig. 3B). ERK phosphorylation was $9 \pm 0.1$ - and $9.5 \pm 0.3$-fold higher after 5 and 10 minutes of stimulation with IGF-I, respectively, when the response of RECs grown in $25 \mathrm{mM}$ glucose was compared with the response of cells grown in $5 \mathrm{mM}(n=3)$.

To demonstrate that changes in $\alpha \mathrm{V} \beta 3$ ligand occupancyin particular binding of the Vn HBD to the C-loop region of $\beta 3$ - could mediate the difference in Shc phosphorylation, we determined whether the Vn HBD directly regulated the ability of IGF-I to activate these signaling intermediaries. In the absence of $\alpha \mathrm{V} \beta 3$ ligand occupancy, IGF-I was unable to stimulate a significant increase in Shc phosphorylation (a $1.6 \pm 0.08$-fold increase; $n=3$; Fig. 3A). However, when RECs were preincubated with the Vn HBD before the addition of IGF-I, a significant ( $6.7 \pm 2$-fold; $n=3$ ) increase in Shc phosphorylation was detected (Fig. 3A). Similarly, when the cells were pretreated with the Vn HBD, IGF-I stimulated a significant $(9.15 \pm 5.8 ; n=3)$ increase in ERK phosphorylation (Fig. 3D).

To demonstrate that the effect of the Vn HBD on IGF-Istimulated Shc phosphorylation was due to $\beta 3$ ligand occupancy, we examined Shc phosphorylation in the presence of the C-loop $\beta 3$ antibody. Blocking the binding of Vn HBD to $\beta 3$ blocked the enhancing effect of Vn HBD on IGF-I-stimulated Shc phosphorylation (Fig. 3E). Control IgG had no effect (data not shown).
The small but nonsignificant increase in Shc phosphorylation in the presence of the Vn HBD alone is consistent with the small but nonsignificant direct effect of the Vn HBD on cell proliferation. The small increase in basal Shc phosphorylation observed when RECs were grown in $25 \mathrm{mM}$ glucose is also consistent with the idea that increased $\alpha \mathrm{V} \beta 3$ ligand occupancy alone is sufficient to stimulate Shc phosphorylation in the absence of exogenously added IGF-I.

\section{Hyperglycemia Regulation of Shc Recruitment to SHPS-1}

We have shown previously that for the cytoplasmic protein Shc to be phosphorylated and activate MAPK it must be recruited to the membrane via its recruitment to phosphorylated tyrosines within the cytoplasmic domain of the transmembrane protein SHPS-1. ${ }^{27}$ We therefore compared SHPS-1 phosphorylation in RECs grown in 5 and $25 \mathrm{mM}$ glucose (Fig. 4). In contrast to the $1.1 \pm 0.2$-fold $(n=3)$ increase in SHPS-1 phosphorylation when RECs grown in $5 \mathrm{mM}$ glucose were exposed to IGF-I, there was a significant $(24 \pm 0.7, n=3)$ increase in the ability of IGF-I to stimulate SHPS-1 phosphorylation when RECs were grown in $25 \mathrm{mM}$ glucose (Fig. 4A). Consistent with the increase in SHPS-1 phosphorylation there was a significant increase in the amount of Shc recruited to SHPS-1 (Fig. 4B) when RECs were grown in $25 \mathrm{mM}$ glucose compared with $5 \mathrm{mM}$ glucose (a $16.3 \pm 6$-fold increase compared with a $1.9 \pm 0.2$-fold increase, $n=3$ ).

The addition of the Vn HBD peptide was sufficient to permit IGF-I to stimulate a significant (a $2.5 \pm 0.08$-fold increase; $n=$ 3 ) increase in SHPS-1 phosphorylation in response to IGF-I (Fig. 4C). Consistent with these results, the addition of the Vn HBD to RECs grown in $5 \mathrm{mM}$ glucose was sufficient to permit IGF-I to stimulate a significant increase (a $2.4 \pm 0.05$-fold, $n=3$ ) in Shc recruitment to SHPS-1 in response to IGF-I (Fig. 4D). The increase in Shc recruitment to SHPS-1 in response to IGF-I in the presence of the Vn HBD was blocked by addition of the C-loop $\beta 3$ antibody (Fig. 4E).

\section{Hyperglycemia Regulation of IAP Association with SHPS-1}

For SHPS-1 to be phosphorylated in response to IGF-I, its extracellular domain must be associated with the extracellular domain of another transmembrane protein IAP. ${ }^{28}$ Given the difference in SHPS-1 phosphorylation in the 5 and $25 \mathrm{mM}$ glucose conditions, we compared the association between SHPS-1 and IAP in the two different conditions (Fig. 5A). IAP association with SHPS-1 was $6.9 \pm 1$.4-fold higher in RECs grown in $25 \mathrm{mM}$ glucose compared with RECs grown in $5 \mathrm{mM}$ glucose $(n=3)$. To demonstrate the ability of glucose to directly regulate the association between IAP and SHPS-1, RECs that had been grown in medium containing $5 \mathrm{mM}$ glucose were incubated for various lengths of time in serum-free medium containing $25 \mathrm{mM}$ glucose. A significant increase in IAP association with SHPS-1 was apparent after 6 hours' incubation with $25 \mathrm{mM}$ glucose (Fig. 5B).

\section{Effect of Blocking IAP Association with SHPS-1 on Shc Phosphorylation and Cell Proliferation in Response to IGF-I}

The anti-IAP antibody B6H12 blocks the association between IAP and SHPS-1. ${ }^{28}$ To demonstrate the significance of the glucose-induced increase in IAP association with SHPS-1, we examined Shc phosphorylation and cell proliferation in RECs grown in $25 \mathrm{mM}$ glucose in the presence of this antibody (Fig. 
A

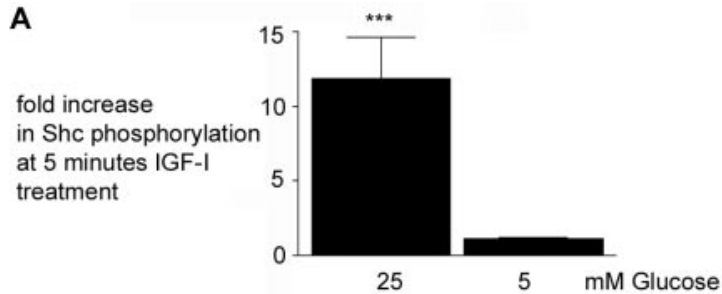

B

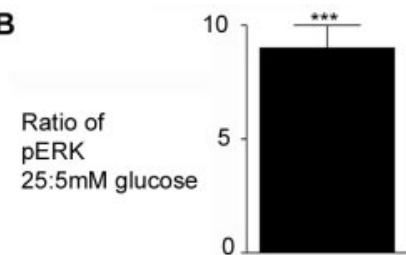

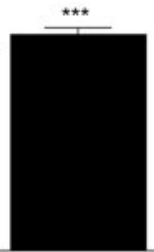

10 (mins) IGF-I
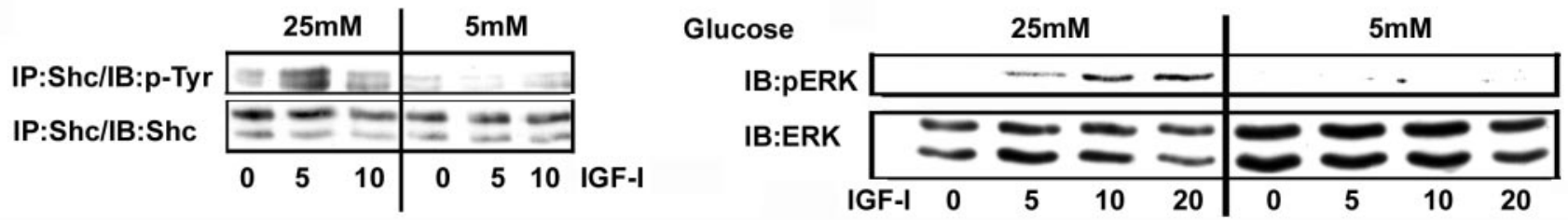

C

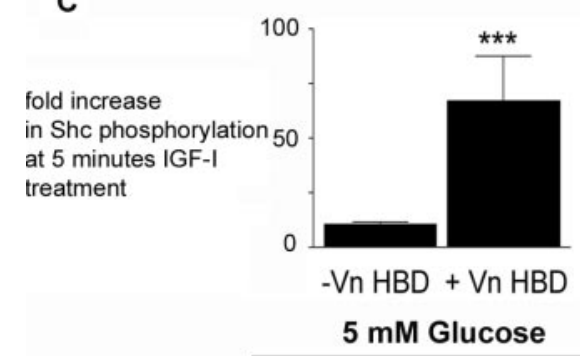

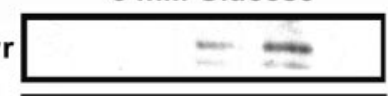

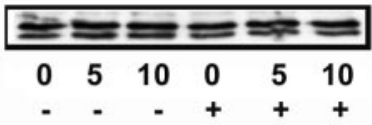

D

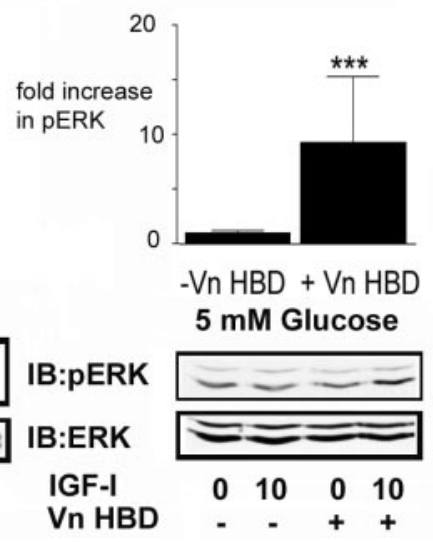

E

FIGURE 3. Glucose regulation of Shc phosphorylation and MAPK activation in response to IGF-I. RECs grown in medium containing either 25 or $5 \mathrm{mM}$ glucose on plates coated with $50 \mu \mathrm{g} / \mathrm{mL}$ fibronectin were quiesced overnight in serum-free medium, and IGF-I (100ng/mL) was added for the lengths of times indicated. Where indicated cells were pretreated with the Vn heparin-binding domain peptide (Vn HBD; $10 \mu \mathrm{g} / \mathrm{mL})$ for $2 \mathrm{hours}$, and then IGF-I $(100 \mathrm{ng} / \mathrm{mL})$ was added for the lengths of times indicated. (A) The extent of Shc phosphorylation was determined by immunoprecipitating cell lysates with an anti-Shc antibody and then immunoblotting with an anti-phosphotyrosine antibody (p-Tyr). Membranes were then stripped and reprobed with an anti-Shc antibody to demonstrate that there was no difference in the amount of Shc that was precipitated in each sample that would account for the difference in Shc phosphorylation. The graph shows the mean increase in Shc phosphorylation $(n=$ 3 independent experiments) after 5 minutes stimulation with IGF-I (**** $P<0.005$ when the increase in response to IGF-I in the cells grown in 25 $\mathrm{mM}$ glucose is compared with the response of cells grown in $5 \mathrm{mM}$ glucose). (B) After cell lysis aliquots containing equal amounts of cell lysate were separated directly by SDS-PAGE and immunoblotted with an antibody specific for phosphorylation of threonine 202 and tyrosine 204 of ERK (top) and total ERK (bottom). The graph shows the ratio of ERK 1 phosphorylation between RECs grown in $25 \mathrm{mM}$ glucose and those grown in $5 \mathrm{mM}$ glucose after 5 and 10 minutes stimulation with IGF-I $\left(n=3\right.$ independent experiments). ${ }^{* * *} P<0.005$ when the increase in response to IGF-I in cells grown in $25 \mathrm{mM}$ glucose is compared to the response of cells grown in $5 \mathrm{mM}$ glucose. (C) The extent of Shc phosphorylation was determined by immunoprecipitating cell lysates with an anti-Shc antibody and immunoblotting with an anti-phosphotyrosine antibody (p-Tyr). Membranes were then stripped and reprobed with an anti-Shc antibody to demonstrate that there was no difference in the amount of Shc that was precipitated in each sample that would account for the difference in Shc phosphorylation. The graph shows the mean increase in Shc phosphorylation ( $n=3$ independent experiments) after 5 minutes; stimulation with IGF-I $(* * * P<0.005$ when the increase in response to IGF-I in the presence of Vn HBD is compared with the response to IGF-I alone). (D) Cells were grown to confluence before overnight incubation in serum free medium. IGF-I $(100 \mathrm{ng} / \mathrm{mL})$ was added for the times indicated. After cell lysis aliquots containing equal amounts of cell lysate were separated directly by SDS-PAGE and immunoblotted with an antibody specific for phosphorylation of threonine 202 and tyrosine 204 of ERK (top) and total ERK (bottom). The graph shows the mean increase in ERK phosphorylation ( $n=3$ independent experiments) after 10 minutes of stimulation with IGF-I (*** $P<0.005$ when the increase in response to IGF-I in the presence of the Vn HBD is compared with the response to IGF-I alone). (E) Cells were treated as in (D) except the C-loop $\beta 3$ antibody (C-loop IgG; $1 \mu \mathrm{g} / \mathrm{mL}$ ) was added to some plates for 2 hours before the addition of the Vn HBD. The extent of Shc phosphorylation was determined by immunoprecipitating cell lysates with an anti-Shc antibody and then immunoblotting with an anti-phosphotyrosine antibody (p-Tyr). Membranes were then stripped and reprobed with an anti-Shc antibody, to demonstrate that there was no difference in the amount of Shc that was precipitated in each sample that would account for the difference in Shc phosphorylation.

6). In the presence of the anti-IAP monoclonal antibody $\mathrm{B} 6 \mathrm{H} 12$ the association between IAP and SHPS-1 was significantly inhibited in RECs grown in $25 \mathrm{mM}$ glucose (Fig. 6A). IAP association with SHPS-1 was reduced by $3.1 \pm 0.2$-fold $(n=3)$ in the presence of the $\mathrm{B} 6 \mathrm{H} 12$ antibody compared with incubation in the presence of the control IgG. Consistent with a role for the association between these two proteins in the regulation of the response of RECs to IGF-I, B6H12 completely inhibited Shc phosphorylation in response to IGF-I (Fig. 6B), and this was associated with a complete inhibition of IGF-I stimulated cell proliferation (Fig. 6C). Cell proliferation in response to IGF-I was reduced from a $1.9 \pm 0.3$-fold increase in the presence of the control IgG, to a $0.8 \pm 0.1$-fold increase in the presence of the IAP antibody, B6H12 $(n=3)$. 

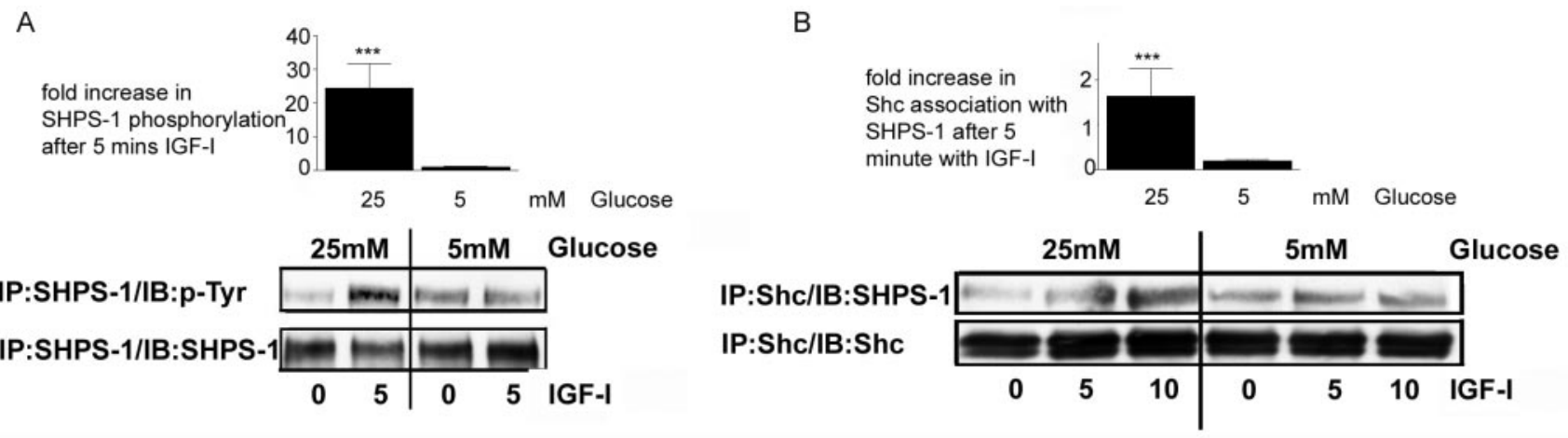
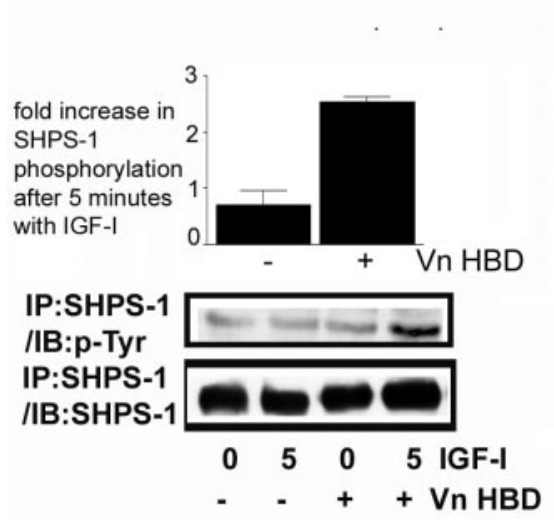
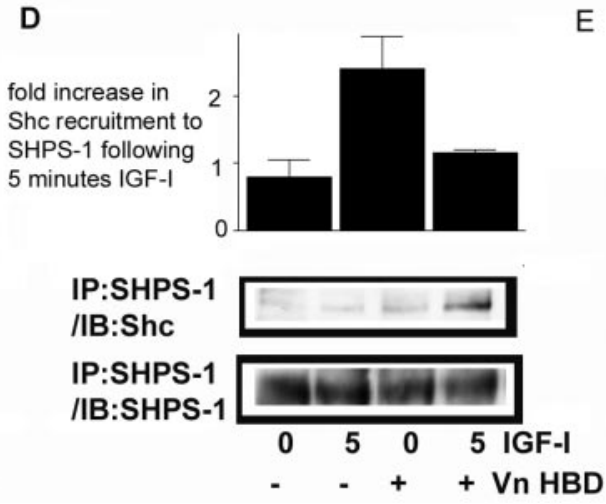

$\mathrm{E}$

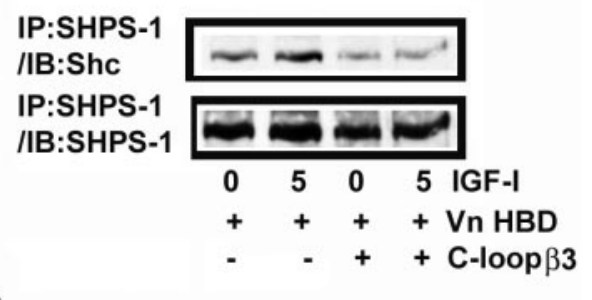

FIGURE 4. Glucose regulation of SHPS-1 phosphorylation and Shc recruitment to SHPS-1 in response to IGF-I. RECs grown in medium containing either 25 or $5 \mathrm{mM}$ glucose on plates coated with $50 \mu \mathrm{g} / \mathrm{mL}$ fibronectin were quiesced overnight in serum-free medium, and then IGF-I (100 ng/mL) was added for the lengths of times indicated. Where indicated, cells were pretreated with Vn heparin-binding domain peptide (Vn HBD; $10 \mu \mathrm{g} / \mathrm{mL})$ for 2 hours, and then IGF-I (100 ng/mL) was added for the lengths of times indicated. (A) The extent of SHPS-1 phosphorylation was determined by immunoprecipitating cell lysates with an anti-SHPS-1 antibody, and then immunoblot analysis was performed with an anti-phosphotyrosine antibody (p-Tyr). Membranes were then stripped and reprobed with an anti-SHPS-1 antibody to demonstrate that there was no difference in the amount of SHPS-1 that was precipitated in each sample that would account for the difference in SHPS-1 phosphorylation. The graph shows the mean increase in SHPS-1 phosphorylation ( $n=3$ independent experiments) after a 5-minute stimulation with IGF-I (*** $P<0.005$ when the increase in response to IGF-I in the cells grown in $25 \mathrm{mM}$ glucose is compared with the response of cells grown in $5 \mathrm{mM}$ glucose). (B) Shc association with SHPS-1 was determined by immunoprecipitating cell lysates with an anti-SHPS-1 antibody and immunoblotting with an anti-Shc antibody. Membranes were then stripped and reprobed with an anti-Shc antibody, to demonstrate that there was no difference in the amount of SHPS- 1 that was precipitated in each sample that would account for the difference in association. The graph shows the mean increase in SHPS-1 association ( $n=3$ independent experiments) after 5 minutes stimulation with IGF-I ( ${ }^{* * *} P<0.005$ when the increase in response to IGF-I in the cells grown in $25 \mathrm{mM}$ glucose is compared with the response of cells grown in $5 \mathrm{mM}$ glucose). (C) The extent of SHPS-1 phosphorylation was determined by immunoprecipitating cell lysates with an anti-SHPS-1 antibody and then immunoblotting with an anti-phosphotyrosine antibody (p-Tyr). Membranes were then stripped and reprobed with an anti-SHPS-1 antibody to demonstrate that there was no difference in the amount of SHPS-1 that was precipitated in each sample that would account for the difference in SHPS-1 association. The graphs show the mean increase in SHPS-1 phosphorylation ( $n=3$, independent experiments) after a 5 -minute stimulation with IGF-I (**** $P<0.005$ when the increase in response to IGF-I in the presence of Vn HBD is compared with the response to IGF-I alone). (D) The extent of Shc association with SHPS-1 was determined by immunoprecipitating cell lysates with an anti-SHPS-1 antibody and then immunoblotting with an anti-Shc antibody. Membranes were then stripped and reprobed with an anti-SHPS-1 antibody, to demonstrate that there was no difference in the amount of SHPS-1 that was precipitated in each sample that would account for the difference in Shc association. The graph shows the mean increase in Shc association ( $n=3$ independent experiments) after a 5-minute stimulation with IGF-I (*** $P<0.005$ when the increase in response to IGF-I in the presence of Vn HBD is compared with the response to IGF-I alone). (B) Cells were treated as for (D) except the C-loop $\beta 3$ antibody (C-loop IgG; $1 \mu \mathrm{g} / \mathrm{mL}$ ) was added to some plates for 2 hours before the addition of the Vn HBD. The graph shows the mean increase in Shc association ( $n=3$ independent experiments) after a 5 -minute stimulation with IGF-I ${ }^{* * *} P<0.005$ when the increase in response to IGF-I in the presence of Vn HBD and C-loop antibody is compared with the response in the presence of the $\mathrm{Vn} \mathrm{HBD}$ alone).

\section{Regulation of IAP Association with SHPS-1 by $\beta 3$ Ligand Occupancy}

To determine whether $\beta 3$ ligand occupancy regulates the association between IAP and SHPS-1 and thereby the response of RECs to IGF-I, we examined the effect of blocking Vn HBD binding to $\beta 3$ on the association of IAP with SHPS-1 (Fig. 7). In the presence of the C-loop $\beta 3$ antibody the association between IAP and SHPS- 1 was reduced by $4.8 \pm$ 0.6-fold $(n=3)$.

\section{Discussion}

Various studies have implicated both IGF-I ${ }^{29-34}$ and the $\alpha \mathrm{V} \beta 3^{7-9}$ as contributors to the retinal neovascularization associated with PDR. The major finding from this study was that culturing RECs in hyperglycemic conditions was associated with an increase in $\beta 3$ ligand binding leading to an increase in its activation state, as measured by its tyrosine phosphorylation, contributing to an enhanced responsiveness of RECs to IGF-I. The role of $\alpha \mathrm{V} \beta 3$ ligand occupancy in regulating the 
A

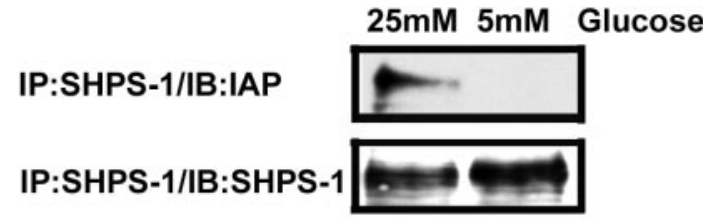

B $5 \rightarrow 25 \mathrm{mM}$ Glucose

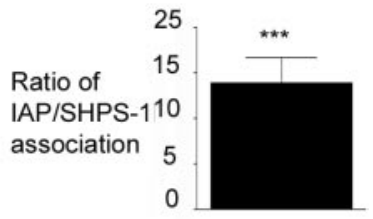

$25: 5 \mathrm{mM}$

FigURE 5. Glucose regulation of IAP association with SHPS-1. (A) RECs grown in medium containing either 25 or $5 \mathrm{mM}$ glucose on plates coated with $50 \mu \mathrm{g} / \mathrm{mL}$ fibronectin were quiesced overnight in serum-free medium. The association of IAP with SHPS- 1 was determined by immunoprecipitating cell lysates with an antiSHPS-1 antibody and then immunoblotting with an anti-IAP antibody. Membranes were then stripped and reprobed with an anti SHPS-1 antibody to demonstrate that there was no difference in the amount of

$$
\begin{array}{lllll}
0 & 2 & 4 & 6 & 16
\end{array} \text { hours of } 25 \mathrm{mM} \text { glucose }
$$
SHPS-1 that was precipitated in each sample that would account for the difference in SHPS-1 phosphorylation. The graph shows the ratio of SHPS-1 association with IAP ( $n=3$ independent experiments) in the cells grown in $25 \mathrm{mM}$ glucose compared with cells grown in $5 \mathrm{mM}$ glucose. ${ }^{* * * *} P<$ 0.005 when the association between IAP and SHPS-1 in RECs grown in $25 \mathrm{mM}$ glucose is compared with their association in RECs grown in $5 \mathrm{mM}$ glucose. (B) Cells grown in $5 \mathrm{mM}$ glucose were incubated overnight in serum-free medium with increasing concentrations of glucose. The association of IAP with SHPS-1 was determined by immunoprecipitating cell lysates with an anti-SHPS-1 antibody and then immunoblotting with an anti-IAP antibody. Membranes were then stripped and reprobed with an anti-SHPS-1 antibody to demonstrate that there was no difference in the amount of SHPS-1 that was precipitated in each sample that would account for the difference in SHPS-1 association with IAP.

response of RECs under hyperglycemic conditions was demonstrated by showing that specifically blocking ligand binding to the C-loop region of $\beta 3$ was sufficient to inhibit the hyperglycemia mediated enhancement in IGF-I signaling response.

Our previous studies have shown that the interaction between the Vn HBD and the C-loop region of $\beta 3$ is sufficient to mediate the positive effects of Vn on IGF-I signaling. ${ }^{14,15}$ In this report, we demonstrated a similar role for the Vn HBD in regulating the response of RECs to IGF-I. This role was demonstrated in two ways. First, we showed that blocking the binding of the Vn HBD to the C-loop region of $\beta 3$ was sufficient to inhibit IGF-I stimulated REC proliferation under conditions of hyperglycemia. Second, we showed that the addition of the Vn HBD was sufficient to stimulate IGF-I responsiveness in RECs grown in $5 \mathrm{mM}$ glucose. Expression of $\alpha \mathrm{V} \beta 3$ is a marker of proliferating endothelial cells. In tumor angiogenesis models $\alpha \mathrm{V} \beta 3$ expression was localized to proliferating and not quiescent endothelial cells, and similar observations have been made in the rodent model of hypoxia induced retinal neovascularization. $^{7,10-13,35}$ An increase in $\alpha \mathrm{V} \beta 3$ integrin expression was also detected in retinal tissue from patients with PDR compared with control samples. ${ }^{36,37}$ In addition, $\alpha \mathrm{V} \beta 3$ antagonists have also been shown to inhibit retinal neovascularization in several models. $8,9,35$ These studies suggest that inhibiting $\alpha \mathrm{V} \beta 3$ inhibits neovascularization regardless of the angiogenic stimuli. ${ }^{7,8}$ RGD-based $\alpha \mathrm{V} \beta 3$ antagonists have been largely unsuccessful in the clinic due to significant negative side effects. Our studies suggest that enhanced $\alpha \mathrm{V} \beta 3$ ligand binding in hyperglycemic conditions may contribute to the increase in REC proliferation in PDR. Blocking $\alpha \mathrm{V} \beta 3$ ligand occupancy with the C-loop $\beta 3$ antibody as a mechanism to block IGF-I signaling offers an alternative strategy to specifically block integrin growth factor cooperativity.

Although we focused on regulation of the MAPK pathway in this study, since it appeared regulated by $\alpha \mathrm{V} \beta 3$ ligand occupancy and hyperglycemia, it is likely that more than one signaling pathway is required for the full effects of IGF-I on RECs. Previous studies have shown that IGF-I-stimulate glucose uptake by RECs is dependent on activation of both the PKC and PI-3 kinase pathways. ${ }^{38,39}$ Activation of the PKC pathway was shown to be dependent on MAPK activation. Since activation of MAPK in our study was dependent on $\alpha \mathrm{V} \beta 3$ ligand occupancy it suggests that the stimulation of glucose uptake by IGF-I may also be dependent on $\alpha \mathrm{V} \beta 3$ ligand occupancy. Further studies will be necessary to determine the relative contribution of $\alpha \mathrm{V} \beta 3$ dependent and independent signaling events. Studies have suggested that activation of the IGF-IR is necessary for the response of RECs to vascular endothelial cell growth factor. ${ }^{40} \alpha \mathrm{V} \beta 3$ has been shown in vivo to regulate the vascular response of mice to VEGF. ${ }^{41}$ It will be interesting to determine
A

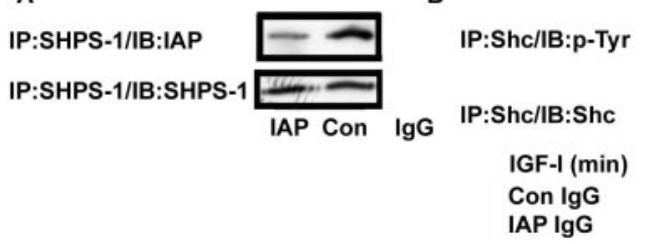

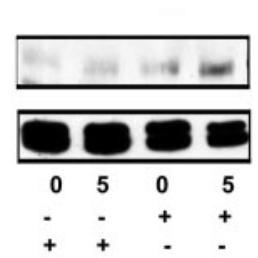

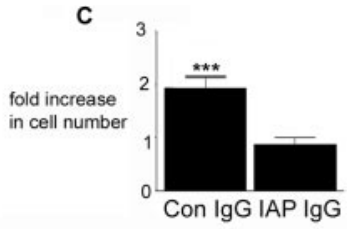

FiguRE 6. Blocking the association between IAP and SHPS-1 inhibits IGFI-mediated signaling in high glucose. RECs grown in medium containing $25 \mathrm{mM}$ glucose on plates coated with $50 \mu \mathrm{g} / \mathrm{mL}$ fibronectin were quiesced overnight in serum-free medium. RECs were then treated with either control IgG (con IgG) or the anti-IAP antibody, B6H12 (IAP IgG), for 4 hours $(10 \mu \mathrm{g} / \mathrm{mL}$ ). (A) The association between IAP with SHPS-1 was determined by immunoprecipitating cell lysates with an anti-SHPS-1 antibody and then immunoblotting with an anti-IAP antibody. Membranes were then stripped and reprobed with an anti-SHPS-1 antibody to demonstrate that there was no difference in the amount of SHPS-1 that was precipitated in each sample that would account for the difference in SHPS-1 association with IAP. The graph shows the ratio of SHPS-1 association with IAP ( $n=3$ independent experiments) between RECs treated with Con IgG and RECs treated with B6H12. ${ }^{*} * *<0.005$ when the association between IAP and SHPS-1 in RECs grown in $25 \mathrm{mM}$ glucose treated with B6H12 is compared with RECs treated with Con IgG. (B) The extent of Shc phosphorylation was determined by immunoprecipitating cell lysates with an anti-Shc antibody and then immunoblotting with an anti-phosphotyrosine antibody (p-Tyr). Membranes were then stripped and reprobed with an anti-Shc antibody to demonstrate that there was no difference in the amount of Shc that was precipitated in each sample that would account for the difference in Shc phosphorylation. (C) The cells $\left(2 \times 10^{4}\right)$ were plated in each well of a 24 -well plate coated with $50 \mu \mathrm{g} / \mathrm{mL}$ of fibronectin and left to attach. The media were then replaced with serum-free media plus $0.2 \%$ FBS plus treatments, as indicated (IGF-I [50 ng/mL], anti IAP antibody B6H12 $[10 \mu \mathrm{g} / \mathrm{mL}]$ or control IgG $[10 \mu \mathrm{g} / \mathrm{mL}]$ ). Forty-eight hours after the addition of IGF-I the number of cells was determined by trypan blue staining and counting. ${ }^{* * * *} P<0.005$ when the increase in cell number in the presence of IGF-I was compared with the increase in cells incubated in the absence of IGF-I. 
FIGURE 7. $\alpha \mathrm{V} \beta 3$ ligand occupancy regulation of the association of IAP with SHPS-1. Cells grown in medium containing either 25 or $5 \mathrm{mM}$ glucose on plates coated with $50 \mu \mathrm{g} / \mathrm{mL} \mathrm{fi}$ bronectin were quiesced overnight in serum-free medium followed by treatment with $1 \mu \mathrm{g} / \mathrm{mL}$ of the Cloop $\beta 3$ antibody (C-loop $\beta 3$ IgG) or control IgG (Con IgG) for 4 hours.

The association between IAP with SHPS-1 was determined by immunoprecipitating cell lysates with an anti-SHPS-1 antibody and then immunoblotting with an anti IAP antibody. The membranes were then stripped and reprobed with an anti-SHPS-1 antibody to demonstrate that there was no difference in the amount of SHPS- 1 that was precipitated. The graph shows the ratio of SHPS-1 association with IAP ( $n=3$ independent experiments) between RECs treated with C-loop $\beta 3$ IgG and control IgG. ${ }^{* * * *} P<0.005$ when the association between IAP and SHPS-1 in RECs grown in $25 \mathrm{mM}$ glucose treated with C-loop $\beta 3$ IgG is compared with RECs treated with Con IgG.

whether the regulation of VEGF signaling by IGF-I also requires $\alpha \mathrm{V} \beta 3$ integrin signaling. When considering the results of this study in the context of those published previously, it is important to note that there is a significant amount of variation in the experimental designs used to study the effects of both glucose and growth factors on REC proliferation (e.g., the use of different substances like gelatin, to coat the tissue culture plates before plating the cells). There is also variation in protocols for transitioning the cells from normal to high glucose medium. Of note, in our study protocols, we plated RECs on fibronectin-coated dishes, cells were given only 4 hours to attach before the proliferation assay commenced, and the cells were transitioned from normal to high glucose containing medium in the presence of serum and allowed 2 to 3 days to adapt before signaling or proliferation assays were examined.

Activation of downstream signaling pathways in response to IGF-I normally requires recruitment of one of two adaptor proteins, either Shc or a member of the IRS family. Recruitment of these adaptor molecules to the cell surface after receptor activation results in their own tyrosine phosphorylation. This effect in turn creates binding sites for the membrane recruitment of Grb-2-Sos which therefore permits activation of the MAPK pathway via activation of Ras. ${ }^{26}$ After IGF-I stimulation, the transmembrane protein SHPS- 1 is phosphorylated, and Shc is recruited to SHPS-1 as a prerequisite for its own tyrosine phosphorylation. ${ }^{27}$ In this study, RECs grown in hyperglycemic conditions demonstrated significantly more SHPS- 1 phosphorylation, Shc recruitment, and subsequent phosphorylation of Shc in response to IGF-I than did RECs grown in normal glucose. The ability of $\alpha \mathrm{V} \beta 3$ ligand occupancy, specifically the interaction between the Vn HBD and the C-loop region of $\beta 3$, to regulate these signaling responses to IGF-I was demonstrated by the enhancement of each of these responses, after the addition of the Vn HBD peptide to RECs grown in normal glucose.

For SHPS-1 to be phosphorylated, it must be bound via its extracellular domain to the extracellular domain of another transmembrane protein, IAP. ${ }^{28}$ The enhanced response of RECs to IGF-I under conditions of hyperglycemia appeared to be attributable to the enhanced association between these two proteins under these conditions. The significance of this interaction was demonstrated by showing the inhibition in IGF-I signaling when the association between these proteins was disrupted with an anti IAP antibody. The role of hyperglycemia in regulating the association between these two proteins, via its effects on $\alpha \mathrm{V} \beta 3$ ligand occupancy, was demonstrated by showing that, when $\beta 3$ ligand occupancy was blocked, IAP association with SHPS-1 was inhibited. The molecular events involved in the hyperglycemia-mediated regulation of $\alpha \mathrm{V} \beta 3$ dependent signaling in RECs in response to IGF-I are summarized in Figure 8.
IAP was identified through its direct physical association with $\alpha \mathrm{V} \beta 3 .{ }^{42}$ To our knowledge, this is the first report of regulation of IAP association with SHPS-1 through changes in $\alpha \mathrm{V} \beta 3$ ligand occupancy. Exactly how $\alpha \mathrm{V} \beta 3$ regulates the association between the two proteins remains to be determined. One possible mechanism is the regulation of IAP conformation by $\alpha \mathrm{V} \beta 3$. It is possible that $\alpha \mathrm{V} \beta 3$ ligand occupancy confers a conformational change on IAP that is necessary for its association with SHPS-1. An alternative possibility is that $\alpha \mathrm{V} \beta 3$ ligand occupancy regulates the membrane distribution of IAP thereby regulating its association with SHPS-1. IAP has been reported to reside in lipid rafts $^{23}$; however, its association with $\alpha \mathrm{V} \beta 3$ requires its movement out of the lipid raft. ${ }^{23}$ Whether this is required for its association with SHPS- 1 and whether this is controlled by ligand occupancy is not known.

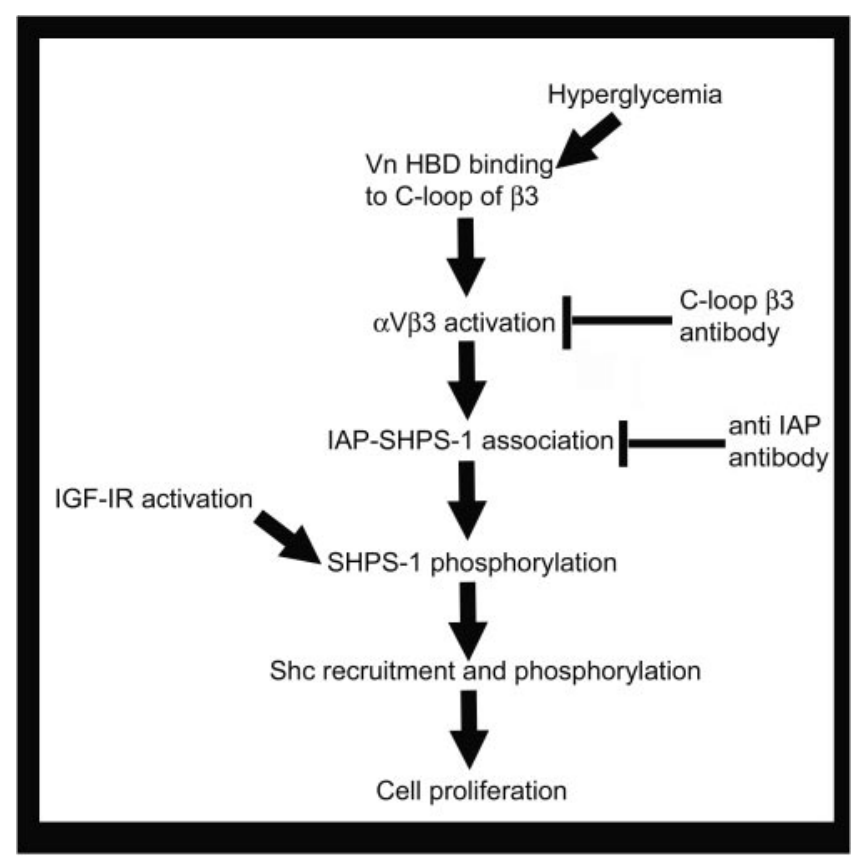

FIGURE 8. Regulation of the response of RECs to IGF-I. The response of RECs to IGF-I requires ligand occupancy of the $\alpha \mathrm{V} \beta 3$ integrin. Hyperglycemia was sufficient to stimulate $\alpha \mathrm{V} \beta 3$ ligand occupancy and thereby enhance the response of RECs to IGF-I. $\alpha \mathrm{V} \beta 3$ ligand occupancy was necessary, to stabilize the interaction between IAP and SHPS-1 which in turn was necessary for SHPS-1 phosphorylation in response to IGF-I. Phosphorylation of SHPS-1 permitted the recruitment and phosphorylation of Shc and therefore downstream signaling in response to IGF-I. Signaling in response to IGF-I was blocked using either an anti- $\alpha \mathrm{V} \beta 3$ antibody (C-loop $\beta 3$ ) or an anti-IAP antibody that disrupts the association between IAP and SHPS-1. 
In summary, our results suggest that one contributing factor to the development of PDR is the hyperglycemia induced activation of the $\alpha \mathrm{V} \beta 3$ integrin as a result of increased ligand occupancy. The consequence of this is enhanced stabilization of the IAP-SHPS-1-Shc signaling complex which, in turn, leads to enhanced signaling in response to IGF-I. Our studies also suggest that antibodies directed against both the C-loop region of $\beta 3$ and IAP may provide novel therapeutic strategies to inhibit the development of PDR. Further studies investigating the effect of both the anti C-loop $\beta 3$ antibody and the anti-IAP antibody in animal models of retinal neovascularization are now needed.

\section{References}

1. Aiello LP, Gardner TW, King GL, et al. Diabetic retinopathy. Diabetes Care. 1998;21:143-156.

2. Schalkwijk CG, Stehouwer CD. Vascular complications in diabetes mellitus: the role of endothelial dysfunction. Clin Sci (Lond). 2005;109:143-159.

3. The Diabetes Control and Complications Trial Research Group. The effect of intensive treatment of diabetes on the development and progression of long-term complications in insulin-dependent diabetes mellitus. $N$ Engl J Med. 1993;329:977-986.

4. Smith LE, Shen W, Perruzzi C, et al. Regulation of vascular endothelial growth factor dependent retinal neovascularization by insulin-like growth factor-1 receptor. Nat Med. 1999;5:1390-1395.

5. Kondo T, Vicent D, Suzuma K, et al. Knockout of insulin and IGF-1 receptors on vascular endothelial cells protects against retinal neovascularization. J Clin Invest. 2003;111:1835-1842.

6. Russell JW, Feldman EL. Insulin-like growth factor-I prevents apoptosis in sympathetic neurons exposed to high glucose. Horm Metab Res. 1999;31:90-96.

7. Friedlander M, Theesfeld CL, Sugita M, et al. Involvement of integrins alpha $\mathrm{v}$ beta 3 and alpha $\mathrm{v}$ beta 5 in ocular neovascular diseases. Proc Natl Acad Sci USA. 1996;93:9764-9769.

8. Hammes HP, Brownlee M, Jonczyk A, Sutter A, Preissner KT. Subcutaneous injection of a cyclic peptide antagonist of vitronectin receptor-type integrins inhibits retinal neovascularization. Nat Med. 1996;2:529-533.

9. Wilkinson-Berka JL, Jones D, Taylor G, et al. SB-267268, a nonpeptidic antagonist of alpha(v)beta 3 and alpha(v)beta5 integrins, reduces angiogenesis and VEGF expression in a mouse model of retinopathy of prematurity. Invest Ophthalmol Vis Sci. 2006;47: $1600-1605$.

10. Brooks PC, Clark RA, Cheresh DA. Requirement of vascular integrin alpha v beta 3 for angiogenesis. Science. 1994;264:569-571.

11. Brooks PC, Montgomery AM, Rosenfeld M, et al. Integrin alpha v beta 3 antagonists promote tumor regression by inducing apoptosis of angiogenic blood vessels. Cell. 1994;79:1157-1164.

12. Brooks PC, Stromblad S, Klemke R, Visscher D, Sarkar FH, Cheresh DA. Antiintegrin alpha $\mathbf{v}$ beta 3 blocks human breast cancer growth and angiogenesis in human skin. J Clin Invest. 1995;96: 1815-1822.

13. Friedlander M, Brooks PC, Shaffer RW, Kincaid CM, Varner JA, Cheresh DA. Definition of two angiogenic pathways by distinct alpha v integrins. Science. 1995;270:1500-1502.

14. Maile LA, Busby WH, Sitko K, et al. The heparin-binding domain of vitronectin is the region that is required to enhance insulin-like growth factor-I signaling. Mol Endocrinol. 2006;20:405-411.

15. Maile LA, Busby WH, Sitko K, et al. IGF-I signaling in smooth muscle cells is regulated by ligand binding to the $177 \mathrm{CYD}$ MKTTC184 sequence of the $\beta 3$ subunit of $\alpha \mathrm{V} \beta 3$. Mol Endocrinol. 2006;20:881-892.

16. Holmes DIR, Wahab NA, Mason RM. Identification of glucoseregulated genes in human mesangial cells by mRNA differential display. Biochem Biophys Res Commun. 1997;238:179-184.

17. Sheibani N, Sorenson CM, Cornelius LA, Frazier WA. Thrombospondin-1, a natural inhibitor of angiogenesis, is present in vitreous and aqueous humor and is modulated by hyperglycemia. Biochem Biophys Res Commun. 2000;267:257-261.
18. Wang S, Skorczewski J, Feng X, Mei L, Murphy-Ullrich JE. Glucose up-regulates thrombospondin 1 gene transcription and transforming growth factor- $\beta$ activity through antagonism of cGMP-dependent protein kinase repression via upstream stimulatory factor 2 . J Biol Chem. 2004;279:34311-34322.

19. Stenina OI, Krukovets I, Wang K, et al. Increased expression of thrombospondin-1 in vessel wall of diabetic Zucker rat. Circulation. 2003;107:3209-3215.

20. Takemoto M, Yokote K, Yamazaki M, et al. Enhanced expression of osteopontin by high glucose: involvement of osteopontin in diabetic macroangiopathy. Ann NY Acad Sci. 2000;902:357-363.

21. Yoon S, Gingras D, Bendayan M. Alterations of vitronectin and its receptor alpha(v) integrin in the rat renal glomerular wall during diabetes. Am J Kidney Dis. 2001;38:1298-1306.

22. Maile LA, Clemmons DR. Integrin-associated protein binding domain of thrombospondin-1 enhances insulin-like growth factor-I receptor signaling in vascular smooth muscle cells. Circ Res. 2003; 93:925-931.

23. Maile LA, Imai Y, Clarke JB, Clemmons DR. Insulin-like growth factor I increases alpha Vbeta 3 affinity by increasing the amount of integrin-associated protein that is associated with non-raft domains of the cellular membrane. J Biol Chem. 2002;277:1800 1805.

24. Zheng B, Clemmons DR. Blocking ligand occupancy of the alphaVbeta3 integrin inhibits insulin- like growth factor I signaling in vascular smooth muscle cells. Proc Natl Acad Sci USA. 1998;95: 11217-11222.

25. Ling Y, Maile LA, Clemmons DR. Tyrosine phosphorylation of the $\beta 3$-subunit of the $\alpha \mathrm{V} \beta 3$ integrin is required for membrane association of the tyrosine phosphatase SHP-2 and its further recruitment to the insulin-like growth factor I receptor. Mol Endocrinol. 2003; $17: 1824-1833$

26. Clemmons DR, Maile LA. Minireview: Integral membrane proteins that function coordinately with the insulin-like growth factor I receptor to regulate intracellular signaling. Endocrinology. 2003; 144:1664-1670.

27. Ling Y, Maile LA, Lieskovska J, Badley-Clarke J, Clemmons DR. Role of SHPS-1 in the regulation of insulin-like growth factor I-stimulated Shc and mitogen-activated protein kinase activation in vascular smooth muscle cells. Mol Biol Cell. 2005;16:3353-3364.

28. Maile LA, Badley-Clarke J, Clemmons DR. The association between integrin-associated protein and SHPS-1 regulates insulin-like growth factor-I receptor signaling in vascular smooth muscle cells. Mol Biol Cell. 2003;14:3519-3528.

29. Grant M, Russell B, Fitzgerald C, Merimee TJ. Insulin-like growth factors in vitreous: studies in control and diabetic subjects with neovascularization. Diabetes. 1986;35:416-420.

30. Pfeiffer A, Spranger J, Meyer-Schwickerath R, Schatz H. Growth factor alterations in advanced diabetic retinopathy: a possible role of blood retina barrier breakdown. Diabetes. 1997;46(suppl 2): S26-S30.

31. Meyer-Schwickerath R, Pfeiffer A, et al. Vitreous levels of the insulin-like growth factors I and II, and the insulin-like growth factor binding proteins 2 and 3 , increase in neovascular eye disease: studies in nondiabetic and diabetic subjects. J Clin Invest. 1993;92:2620 - 2625 .

32. Sonksen PH, Russell-Jones D, Jones RH. Growth hormone and diabetes mellitus: a review of sixty-three years of medical research and a glimpse into the future? Horm Res. 1993;40:68-79.

33. Dills DG, Moss SE, Klein R, Klein BE, Davis M. Is insulinlike growth factor I associated with diabetic retinopathy? Diabetes. 1990;39: 191-195.

34. Dills DG, Moss SE, Klein R, Klein BE. Association of elevated IGF-I levels with increased retinopathy in late-onset diabetes. Diabetes. 1991; $40: 1725-1730$

35. Chavakis E, Riecke B, Lin J, et al. Kinetics of integrin expression in the mouse model of proliferative retinopathy and success of secondary intervention with cyclic RGD peptides. Diabetologia. 2002; 45:262-267.

36. Delannet M, Martin F, Bossy B, Cheresh DA, Reichardt LF, Duband JL. Specific roles of the alpha $\mathrm{V}$ beta 1 , alpha $\mathrm{V}$ beta 3 and alpha $\mathrm{V}$ beta 5 integrins in avian neural crest cell adhesion and migration on vitronectin. Development. 1994;120:2687-2702. 
37. Robbins SG, Brem RB, Wilson DJ, et al. Immunolocalization of integrins in proliferative retinal membranes. Invest Ophthalmol Vis Sci. 1994;35:3475-3485.

38. DeBosch BJ, Deo BK, Kumagai AK. Insulin-like growth factor-1 effects on bovine retinal endothelial cell glucose transport: role of MAP kinase. J Neurochem. 2002;81:728-734.

39. DeBosch BJ, Baur E, Deo BK, Hiraoka M, Kumagai AK. Effects of insulin-like growth factor-1 on retinal endothelial cell glucose transport and proliferation. J Neurochem. 2001;77: 1157-1167.
40. Hellstrom A, Perruzzi C, Ju M, et al. Low IGF-I suppresses VEGFsurvival signaling in retinal endothelial cells: direct correlation with clinical retinopathy of prematurity. Proc Natl Acad Sci USA. 2001;98:5804-5808.

41. Weis SM, Lindquist JN, Barnes LA, et al. Cooperation between VEGF and beta 3 integrin during cardiac vascular development. Blood. 2007;109:1962-1970.

42. Schwartz MA, Brown EJ, Fazeli B. A 50-kDa integrin-associated protein is required for integrin-regulated calcium entry in endothelial cells. J Biol Chem. 1993;268:19931-19934. 